\title{
National Vegetation Database of South Africa
}

\author{
Leslie Ward Powrie, Michael Charles Rutherford, Ladislav Mucina \& Kagiso Mangwale
}

\begin{abstract}
Southern Africa has been recognized as one of the most interesting and important areas of the world from an ecological and evolutionary point of view. The establishment and development of the National Vegetation Database (NVD) of South Africa (GIVD ID AF-ZA-001) has contributed to environmental planning and conservation management in this floristically unique region. The NVD was developed using TURBOVEG and has been moved to MS Access. It currently contains 279 datasets, 47,466 vegetation plots (relevés) sharing 11,759 plant taxa and containing 1,064,100 species occurrence records. More than $80 \%$ of the studies use the BraunBlanquet method. The NVD was primarily founded to serve vegetation classification and mapping goals but has also been an important tool in conservation assessment, target setting, production of the National Vegetation Map, National Forest Type Classification, South African National Biodiversity Assessment, Forest Type Conservation Assessment. However, the use of the current NVD in multidisciplinary research has certainly not been fully explored. Well-trained vegetation surveyors are needed so that the NVD will continue to be purpose driven and serve the needs of biological survey in pursuit of sustainable use of the vegetation and flora resources of the southern African subcontinent.
\end{abstract}

Keywords: climate change; conservation assessment; conservation management; conservation target; cover-abundance gradient; environmental planning; phytosociology; spatial species diversity; species co-occurrence; vegetation classification.

\section{National Vegetation Database of South Africa}

Scope: Phytosociological information mainly from South Africa. There are a few relevés in adjacent countries. Plot based surveys with full species records, relative abundance, georeferencing and plot size are preferred.

Status: ongoing capture

Period: $1968-2010$

Database manager(s): Leslie Ward Powrie (I.powrie@sanbi.org.za); Ladislav Mucina (L.Mucina@curtin.edu.au); Kagiso Mangwale (k.mangwale@sanbi.org.za)

Owner: Hosted at South African National Biodiversity Institute, under the direction of the National Vegetation Map Committee. Contact address: NVMC, Applied Biodiversity Research Division, South African National Biodiversity Institute, Private Bag X7, Claremont, 7735, South Africa. Web address: http://www.sanbi.org.za

Availability: according to a specific agreement

Database format(s):MS Access

Online upload: no

Online search: no problems and future. S Afr J Sci. 2012;108(1/2), Art. \#629, 8 pages. http://dx.doi.org/10.4102/sajs.v108i1/2.629

Plot type(s): normal plots

Non-overlapping plots: 47,466

Plot-size range: $1-400 \mathrm{~m}^{2}$

Total plot observations: 47,466

Estimate of existing plots: 47,466

Number of sources: 486

Completeness: $100 \%$

Countries: BW: 0.3\%; LS: 0.6\%; MZ: 1.3\%; NA: 0.6\%; ZA: $96.8 \%$; ZW: $0.3 \%$

Forest: $10 \%$ - Non-forest: aquatic: $0 \%$; semi-aquatic: $0 \%$; arctic-alpine: $0 \%$; natural: $90 \%$; semi-natural: 0\%; anthropogenic: $0 \%$

Guilds: all vascular plants: $88 \%$; only trees and shrubs: $12 \%$; bryophytes (terricolous or aquatic): $4 \%$; lichens (terricolous or aquatic): $3 \%$; algae (terricolous or aquatic): $0 \%$

Environmental data: altitude: $17 \%$; slope aspect: $6 \%$; slope inclination: $16 \%$; soil depth: $4 \%$; surface cover other than plants (open soil, litter, bare rock etc.): $45 \%$; soil $\mathrm{pH}$ : $1 \%$; other soil attributes: $1 \%$, land use categories: $1 \%$

Performance measure(s): presence/absence only: $2.4 \%$; cover: $97.6 \%$

Geographic localisation: GPS coordinates (precision $25 \mathrm{~m}$ or less): $20 \%$; point coordinates less precise than GPS, up to $1 \mathrm{~km}$ : $10 \%$; small grid (not coarser than $10 \mathrm{~km}): 17 \%$; political units or only on a coarser scale $(>10 \mathrm{~km}): 53 \%$

Sampling periods: $1940-1949: 0.0 \% ; 1950-1959: 0.2 \% ; 1960-1969: 0.2 \% ; 1970-1979: 5.5 \% ; 1980-1989: 13.2 \% ; 1990-1999: 16.0 \% ; 2000-2009$ : $5.3 \%$; unknown $59.5 \%$

Information as of 2012-07-25; further details and future updates available from http://www.givd.info/ID/AF-ZA-001

Leslie Ward Powrie*(1.powrie@sanbi.org.za), Michael Charles Rutherford (m.rutherford@sanbi.org.za), Kagiso Mangwale

(k.mangwale@sanbi.org.za)

South African National Biodiversity Institute (SANBI), Private Bag X7, 7735 Claremont, SOUTH AFRICA

Ladislav Mucina (L.Mucina@curtin.edu.au)

Department of Environment and Agriculture, Curtin University, GPO Box U1987, WA 6845 Perth, AUSTRALIA

*Corresponding author 